\title{
Recent Developments in Solar Energy Harvesting and Photocatalysis
}

\author{
Junwang Tang, ${ }^{1}$ Songyuan Dai, ${ }^{2}$ and Jawwad A. Darr ${ }^{3}$ \\ ${ }^{1}$ Department of Chemical Engineering, University College London, Torrington, Place, London WC1E 7JE, UK \\ ${ }^{2}$ Key Lab of Novel Thin Film Solar Cells, Institute of Plasma Physics, Chinese Academy of Sciences, \\ P.O. Box 1126, Hefei, Anhui, China \\ ${ }^{3}$ Department of Chemistry, University College London, Gordon Street, London WC1H 0AJ, UK \\ Correspondence should be addressed to Junwang Tang, junwang.tang@ucl.ac.uk
}

Received 4 March 2012; Accepted 4 March 2012

Copyright (C) 2012 Junwang Tang et al. This is an open access article distributed under the Creative Commons Attribution License, which permits unrestricted use, distribution, and reproduction in any medium, provided the original work is properly cited.

Development of renewable energy harvesting methods and working towards a sustainable environment are two major challenges facing humanity. One of the most promising solutions to the former challenge encompasses solar energy conversion to fuels or electricity. There are three key processes required for far more efficient solar energy utilisation, including maximising photon absorption, efficient charge separation (minimal recombination) and finally, effective utilisation of these separated charges. This special issue aims to address some of these important points in very different circumstances. Therefore, the papers published in this issue focus on recent developments in device design, concentrated solar energy and improved understanding of photovoltaic modules. This research will provide very useful information for device performance enhancement and practical system implementation.

In detail, the article authored by Subiyanto Subiyanto et al. describes a hopfield neural network optimized fuzzy logic controller for efficient power point tracking in photovoltaic systems. The simulation results demonstrate the proposed device would be efficient in practical applications. In contrast, Lei Jing et al. designed a prototype to more efficiently concentrate solar energy, which illustrates a novel non-imaging optical structure that can be used to improve the aspect ratio, optical efficiency, and uniformity of incident photon flux. The research authored by Huifu Zhao is an excellent report about a two-stage solar concentrator, which is composed of the Fresnel lens and achieves very high concentration efficiency (up to $85 \%$ ). The authors have also verified that the system works equally well whether it is indoors or outdoors. The light divergence angle of the indoor concentration test system is $0.267^{\circ}$ which also matches with the sunlight divergence angle. The indoor concentration test system with large caliber $(145 \mathrm{~mm})$ has shown that such a two-stage structured solar concentrator with relatively low cost is appropriate for broad implementation. The research article by Eladio Durán et al. describes novel I-V/P-V characteristic curves for a PV generator using a controlled DC-DC converter. The main idea of this paper is to use the DC-DC converter as a varying resistor by controlling the duty cycle of its triggering signal. The advantage of the proposed method is that it is capable to test different PV panels with different ratings unlike the capacitor method but being more practical. In the short review by Ramabadran Ramaprabha and Badrilal Mathur, the performance of several different configurations of PV modules, including series-parallel configuration (SP), total cross tied configuration (TCT), bridge linked configuration (BL) and honey-comb configuration (HC) using them in series or parallel was compared. Following systematical analysis, TCT seems the best in terms of power produced and $\mathrm{HC}$ is the second best. A simulation model authored by N. Pandiarajan et al. was developed for photovoltaic power systems by using Matlab/Simulink. The designed model has been validated experimentally to optimise it. The reported photovoltaic devices work more efficiently for solar energy conversion if the solar intensity can be enhanced. Finally, Haixiang Zang et al. proposed a modified typical meteorological year (TMY) method for generating TMY from practical measured weather data. TMYs of 35 cities in China were generated based on the latest and accurate measured weather 
data. Eleven weather indices and novel assigned weighting factors were applied in the research in the processing of forming the TMY database, which is very good reference in the utilizations of solar energy systems, in particular in China.

Apart from content addressed in this issue, the strategy of the advanced materials engineering, concepts on device design and new insights into mechanisms are also very interesting and developing apace. In particular, solar $\mathrm{H}_{2}$ production from water photolysis, $\mathrm{CO}_{2}$ storage or conversion driven by solar energy, advances in the dye-sensitized solar cells, photocatalytic wastewater and gas treatment and nanostructured materials application in the solar energy conversion are important domains in solar energy utilisation and complementary to this issue, which are expected to be addressed in the other issues.

Junwang Tang

Songyuan Dai

Jawwad A. Darr 


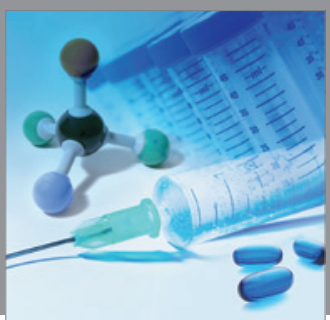

International Journal of

Medicinal Chemistry

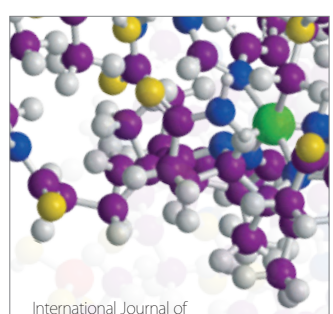

Carbohydrate Chemistry

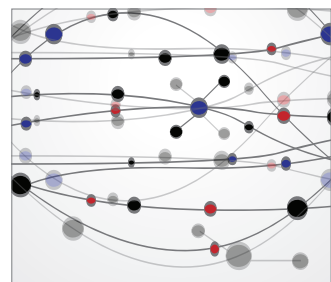

The Scientific World Journal
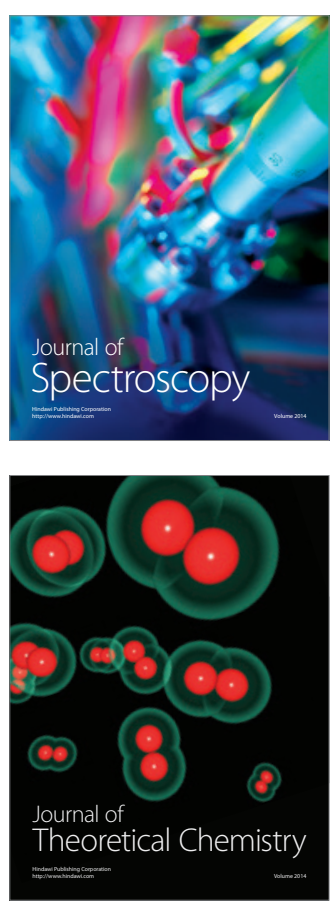
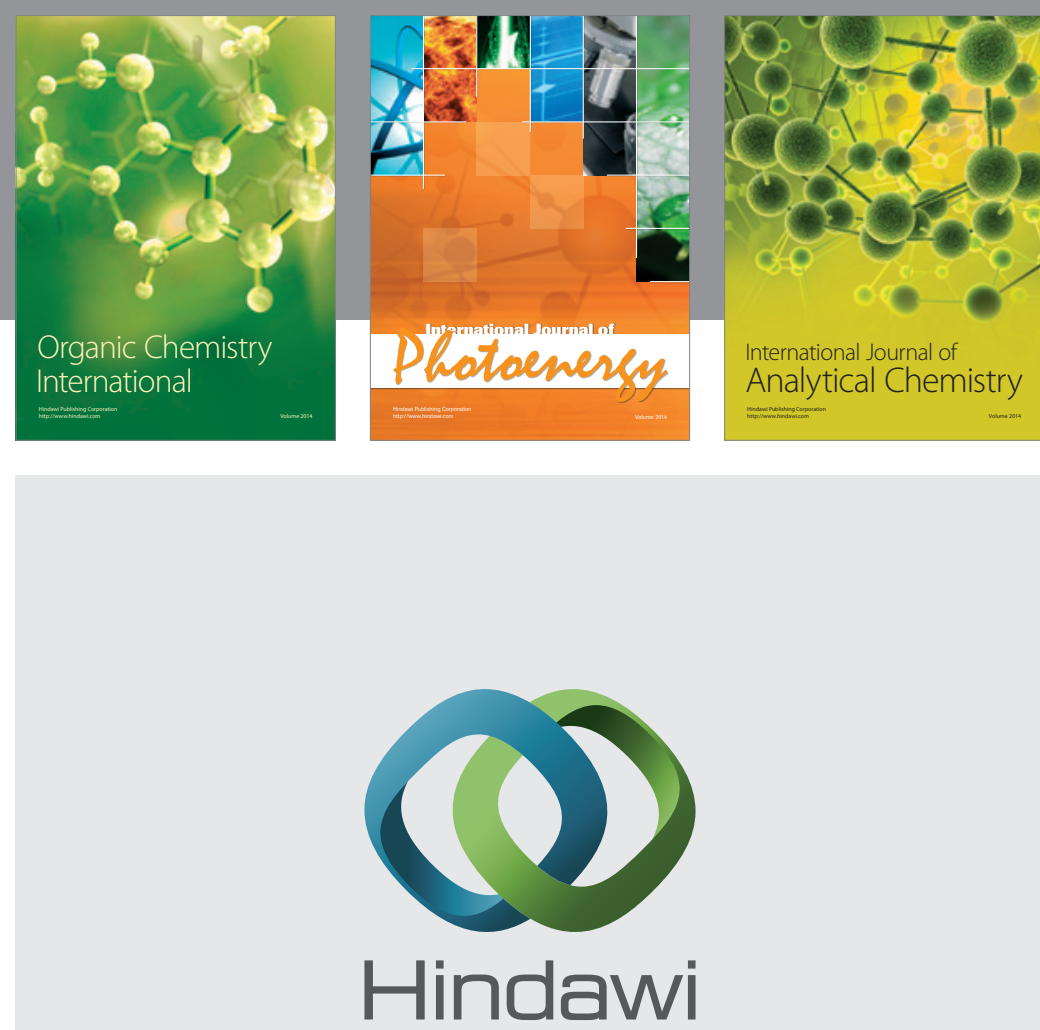

Submit your manuscripts at

http://www.hindawi.com
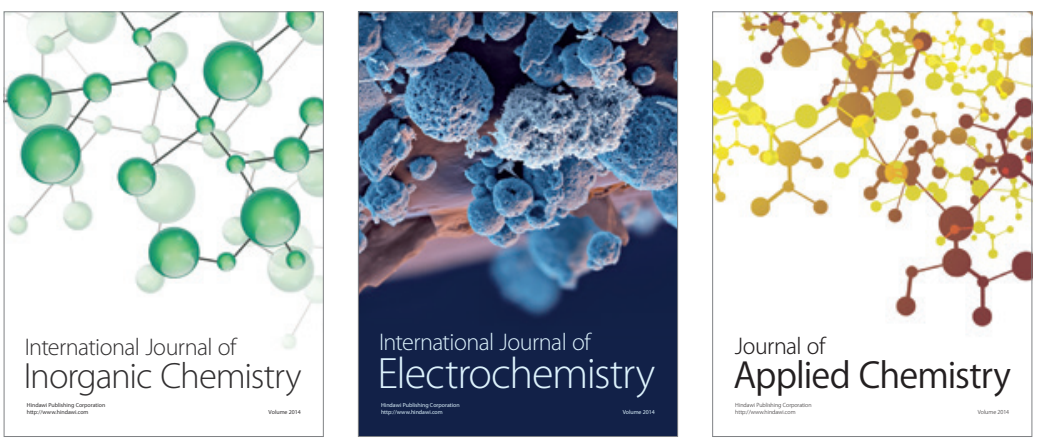

Journal of

Applied Chemistry
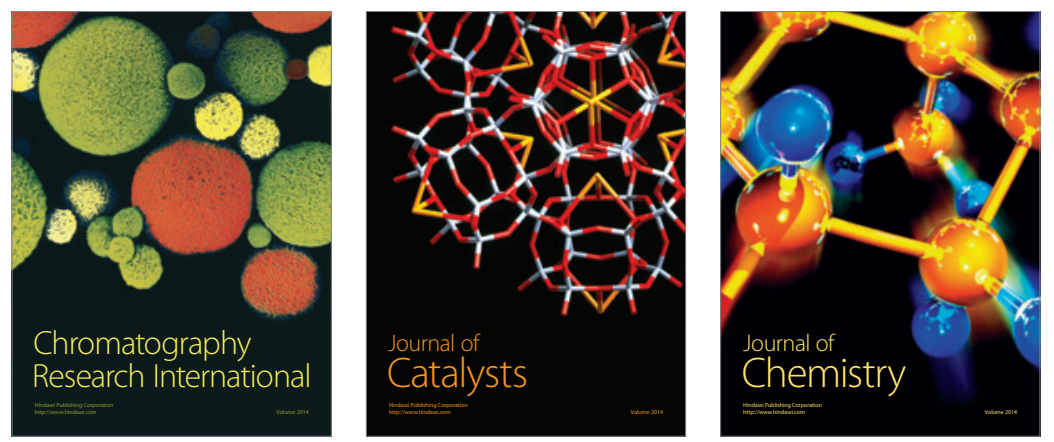
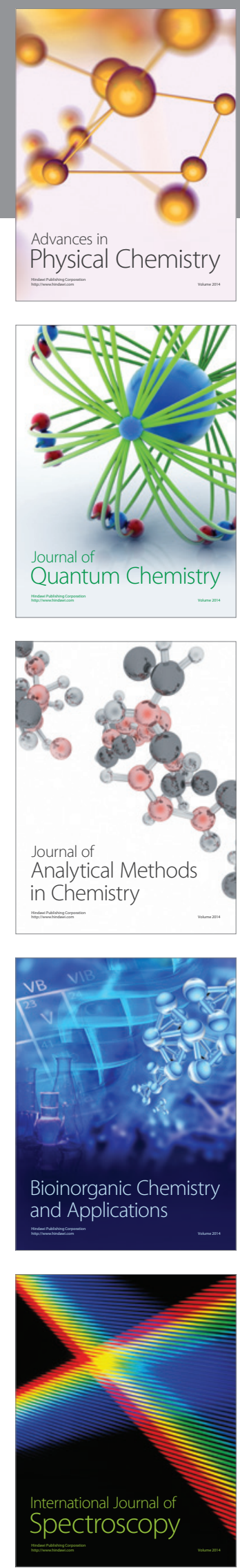